\title{
Controlled Antibiotics Release System through Simple Blended Electrospun Fibers for Sustained Antibacterial Effects
}

\author{
Zixin Zhang ${ }^{a, b}$, Jianxiong Tang $^{c}$, Heran Wang ${ }^{d}$, Qinghua Xia ${ }^{a, b}$, Shanshan \\ $X u^{a *}$, and Charles $C \mathrm{Han}^{a} *$
}

\begin{abstract}
${ }^{a}$ State Key Laboratory of Polymer Physics and Chemistry, Joint Laboratory of Polymer Science and Materials, Beijing National Laboratory for Molecular Sciences, Institute of Chemistry, Chinese Academy of Sciences, Beijing 100190, P. R. China

${ }^{\mathrm{b}}$ University of Chinese Academy of Sciences, Beijing 100049, P. R. China

${ }^{c}$ Huadong Hospital affiliated to Fudan University, Shanghai 200040, P. R. China

${ }^{\mathrm{d}}$ Torch High Technology Industry Development Center, Ministry of Science and Technology, Beijing 100045, P. R. China
\end{abstract}

Corresponding authors:

Charles C Han (email: c.c.han@iccas.ac.cn),

Shanshan Xu (email: xuss04@iccas.ac.cn).

Telephone: +86 82618089. Fax: +86 62521519 


\section{Materials and methods}

\subsection{Materials}

PLGA $(\mathrm{Mw}=60,000, \mathrm{LA} / \mathrm{GA}=50 / 50)$ and PEG- $b$-PDLLA $(\mathrm{Mw}, 5000-b-2500)$ were purchased from Jinan Daigang Biology Engineer Co., Ltd (Shandong, China). PEG (Mw $=6,000$ ), PGA (inherent viscosity $1.4 \mathrm{dL} / \mathrm{g}$ ) and PDO (inherent viscosity $1.4 \sim 2.2 \mathrm{dL} / \mathrm{g}$ ) were purchased from Sigma-Aldrich (MO, USA). Moxifloxacin (Mo) was obtained from Hubei Aidan Pharm\&Chem Co., Ltd (Hubei, China). Moxifloxacin hydrochloride (MoH) was purchased from Jiangsu Haiheng Pharmaceutical Co., Ltd (Jiangsu, China). Ciprofloxacin hydrochloride $(\mathrm{CiH})$ was obtained from Haiheng Pharmaceutical Co., Ltd (Jiangsu, China). Hexafluoroisopropanol (HFIP) was obtained from Jinan Wedo Industrial Co., Ltd (Shandong, China). Phosphate-buffered saline (PBS, pH = 7.2 7.4) was purchased from Sinopharm Chemical Reagent Beijing Co., Ltd (Beijing, China). All of them were used without further purification.

\subsection{Fabrication of drug loaded electrospun membranes}

PLGA and PDO (or PEG-b-PDLLA, PGA) solution with a ratio of 93/7 w/w was prepared in HFIP, and the total concentration was $25 \mathrm{w} / \mathrm{v} \%$ ( $\mathrm{w}$ in $\mathrm{g}$ and $\mathrm{v}$ in $\mathrm{mL}$ ). Then, one of model drugs $(\mathrm{Mo}, \mathrm{MoH}$ or $\mathrm{CiH})$ were dissolved in polymer solutions with a concentration of $10 \mathrm{w} / \mathrm{w} \%$ of total polymer content. The electrospinning process was carried out in a sterile environment at $20 \mathrm{kV}$ and a steady flow rate of $20 \mu \mathrm{L} / \mathrm{min}$. the distance between the spinneret and the collector was set as $17 \mathrm{~cm}$. the fabricated electrospun membranes were dried under vacuum at room temperature for 2 days prior to usage. 


\subsection{In vitro drug release}

The dried electrospun membranes were cut into pieces with a weight of $10 \mathrm{mg}$, and each of them was placed in an individual vial containing $10 \mathrm{~mL}$ PBS. All of the vials were incubated in a vapor-bathing constant temperature vibrator at $37^{\circ} \mathrm{C}$ with a vibrating speed of $100 \mathrm{rpm}$. At specified time intervals, $1 \mathrm{~mL}$ solution of release medium was taken out from each vial and equal volume of fresh PBS replenished. The amount of drug released was determined using a UV-Vis spectrophotometer (PGeneral, China). The accumulated release of drugs was calculated based on a standard calibration curve of each drug. All experiments were performed in quintuplicate, and the data were expressed as the mean \pm standard deviation.

\subsection{Characterization}

1.4.1. Scanning electron microscopy. The surface and cross-section morphologies of the electrospun fibrous films were observed using a scanning electron microscope (SEM) (JEOL, JSM-6700F, Japan). The samples were vacuum sputter coated with platinum before SEM observations.

1.4.2. Confocal laser scanning microscopy. All of $\mathrm{Mo}, \mathrm{MoH}$ and $\mathrm{CiH}$ could be detected by confocal laser scanning microscope. Thus, the distribution of drugs in the electrospun fibers were observed by a confocal laser scanning microscope (CLSM, Olympus, Japan).

\subsection{Antibacterial assessments.}

Staphylococcus aureus (S. aureus, Gram-positive) and Eschericha coli (E. coli, Gramnegative) were used as model bacteria to evaluate the antibacterial activity of electrospun membranes with various composition. Activated $S$. aureus and E. coli with an OD value 
of about 0.5 at $600 \mathrm{~nm}$ were prepared first. For quantitative analysis, $100 \mu \mathrm{L}$ bacteria LB solution was added to each sterile EP tubes with $5 \mathrm{~mL}$ fresh LB medium. Then, $1 \mathrm{mg}$ membranes with or without drugs were introduced into each tube. All test tubes were incubated at $37^{\circ} \mathrm{C}$ with a vibrating speed of $150 \mathrm{rpm}$. After 24 hours, $100 \mu \mathrm{L}$ solution of each tubes were spreaded onto agar plates. After incubating at $37{ }^{\circ} \mathrm{C}$ for $24 \mathrm{~h}$ without vibration, the resulting bacterial colonies on the plates were counted visually. The antibacterial rate were calculated using the difference between the number of colonies of test samples and the control.

For the inhibition zone test, $100 \mu \mathrm{L}$ bacterial LB solution were first spreaded onto the agar plates, and then circular membranes with a diameter of about $1 \mathrm{~cm}$ and the same mass were pasted onto the plates. After incubating at $37{ }^{\circ} \mathrm{C}$ for $24 \mathrm{~h}$, the inhibition diameter (the difference between the outer diameter and the inner diameter) of membranes were counted. All experiments were performed in triplicate.

To investigate the antibacterial activity of membranes after specified period of drug release, membranes after incubating in PBS for 3 or 9 days drug release were also tested under the same conditions.

1.6. Statistical analysis. Data in this study were expressed as mean \pm standard deviation (SD). Whenever appropriate, two-tailed Student's $t$-test was used to discern the statistical difference between groups. A probability value $(p)<0.05$ was considered to be statistically significant. 